\title{
Multiresolution Analysis with Non-Nested Spaces
}

\author{
Georges-Pierre Bonneau*
}

\begin{abstract}
Two multiresolution analyses (MRA) intended to be used in scientific visualization, and that are both based on a non-nested set of approximating spaces, are presented. The notion of approximated refinement is introduced to deal with non nested spaces. The first MRA scheme, referred to as BLaC (Blending of Linear and Constant) wavelets is based on a one parameter family of wavelet bases that realizes a blend between the Haar and the linear wavelet bases. The approximated refinement is applied in the last part to build a second MRA scheme for data defined on an arbitrary planar triangular mesh.
\end{abstract}

\section{INTRODUCTION}

The purpose of this paper is to present two multiresolution analyses (MRA) intended to be used in scientific visualization, and that are both based on a non-nested set of approximating spaces. The need for non nested spaces arises from the fact that the required scaling functions do not fulfill any refinement equation. Therefore we introduce in the first part the concept of approximated refinement equation, that allows to generalize the filter bank and exact reconstruction algorithms. The second part shows how this concept enables to define a one parameter family of wavelet bases that realizes a blend between the Haar and the linear wavelet bases. These so-called BLaC (Blending of Linear and Constant) wavelets are used for the analysis of volume data defined on uniform grids. The effect of the blending parameter is shown on examples. In the last part of the paper, the approximated refinement is applied to built a MRA scheme for data defined on an arbitrary planar triangular mesh. The ability to deal with arbitrary triangular meshes, without subdivision connectivity, can be achieved only through the use of non nested approximating spaces, as introduced in the first part.

\section{Multiresolution analysis with non nested approximating spaces}

We assume that the reader is familiar with the basic concepts of multiresolution analysis. An introduction on this topic can be found in [4]. In this section, a general framework for constructing multiresolution analysis schemes based on a non nested sequence of approximating spaces is given.

* Laboratoire LMC-IMAG, University of Grenoble, B.P. 53, F-38041 Grenoble cedex 9, France, Tel: +33476514560, Fax +334766312 63, Georges-Pierre.Bonneau@imag.fr 


\subsection{Necessary conditions on the sequence of approximating spaces}

The minimal requirements for this construction are the following: If $L_{2}(\Omega)$ is the set of all functions with finite energy defined on a domain $\Omega$, the sequence $V_{n}$ of approximating subspaces of $L_{2}(\Omega)$ must satisfy:

$$
\begin{aligned}
& V_{n} \text { is isomorph to a subspace of } V_{n+1} \\
& \bigcup V_{n} \text { is dense in } L_{2}(\Omega) \text {. }
\end{aligned}
$$

Condition (1) means that the sequence of approximating spaces $V_{n}$ is "growing" in $L_{2}(\Omega)$. This condition replaces the nested condition $V_{n} \subset V_{n+1}$ in the definition of the classical multiresolution analysis schemes. If the spaces $V_{n}$ have finite dimensions, this condition may be written as $\operatorname{dim} V_{n} \leq \operatorname{dim} V_{n+1}$.

Condition (2) implies that every function can be approximated with an arbitrary precision by functions in $V_{n}$ for some value $n$.

\subsection{Approximated refinement equation}

Let $\left(\varphi_{j}^{n}\right)$ denote the scaling functions (i.e. $\left(\varphi_{j}^{n}\right)_{j}$ is a basis of $\left.V_{n}\right)$. If the nested condition $V_{n} \subset V_{n+1}$ is satisfied, the link between two successive levels of resolution is provided by a so-called refinement equation, which expresses each scaling function $\varphi_{j}^{n}$ as a linear combination of the finer scaling functions $\left(\varphi_{i}^{n+1}\right)$. In our setting, condition (1) implies that there exists functions $\left(\tilde{\varphi}_{j}^{n}\right)_{j}$ which form a basis of a space $\tilde{V}_{n}$, included in $V_{n+1}$. And the link between level $n$ and $n+1$ is now provided through a two step process: First apply the isomorphism, i.e. replace the scaling functions $\left(\varphi_{j}^{n}\right)_{j}$ by the intermediate functions $\left(\tilde{\varphi}_{j}^{n}\right)_{j}$, and then apply the refinement equation which expresses $\tilde{\varphi}_{j}^{n}$ as a linear combination of the finer scaling functions $\left(\varphi_{i}^{n+1}\right)_{i}$ :

$$
\varphi_{j}^{n} \quad \leftrightarrow \quad \tilde{\varphi}_{j}^{n}=\sum_{i} p_{i j}^{n} \varphi_{i}^{n+1}
$$

(3) is called the approximated refinement equation because in the two next sections, where the framework is applied, $\tilde{\varphi}_{j}^{n}$ is closed to $\varphi_{j}^{n}$.

\subsection{Detail spaces and wavelet functions}

The detail spaces $W_{n}$ are now defined as a complementary of the space $\tilde{V}_{n}$ in $V_{n+1}$ :

$$
V_{n+1}=\tilde{V}_{n} \oplus W_{n}
$$

The wavelet functions $\left(\psi_{j}^{n}\right)_{j}$ are basis functions of the space $W_{n}$. Thus there exist coefficients $\left(q_{i j}^{n}\right)$ such that

$$
\psi_{j}^{n}=\sum_{i} q_{i j}^{n} \varphi_{i}^{n+1},
$$

and coefficients $\left(a_{i j}^{n}\right),\left(b_{i j}^{n}\right)$ such that

$$
\varphi_{j}^{n+1}=\sum_{i} a_{i j}^{n} \tilde{\varphi}_{i}^{n}+\sum_{i} b_{i j}^{n} \psi_{i}^{n} .
$$

From now, we assume that the approximating spaces $V_{n}$ have finite dimensions. Let us call $P_{n}, Q_{n}, A_{n}$ and $B_{n}$ the matrices with coefficients $\left(p_{i j}^{n}\right),\left(q_{i j}^{n}\right),\left(a_{i j}^{n}\right)$ and $\left(b_{i j}^{n}\right) . P, Q$ are called synthesis matrices and $A, B$ are called analysis matrices. The columns of the matrices $P$ and $Q$ contain the coordinates of the intermediate functions $\tilde{\varphi}_{j}^{n}$ and the wavelets $\psi_{j}^{n}$ in the basis of the finer scaling functions $\varphi_{i}^{n+1}$. The rows of the matrices $A$ and $B$ contain the coordinates of the dual of the intermediate functions, and the dual of the wavelets, in the dual basis of the finer scaling functions. These matrices must satisfy the reconstruction condition

$$
P A+Q B=I .
$$


Furthermore let $G_{n+1}$ be the Gram-Schmidt matrix of the basis $\left(\varphi_{i}^{n+1}\right)$ (i.e. the matrix whose elements are $<\varphi_{i}^{n+1}, \varphi_{j}^{n+1}>$ ).

Then the multiresolution analysis is called

- semi-orthogonal iff

$$
P^{T} G_{n+1} Q=0, \quad A G_{n+1}^{-1} B^{T}=0 .
$$

In this case $P$ and $Q$ can be calculated respectively from $A$ and $B$ by

$$
P=G_{n+1}^{-1} A^{T}\left(A G_{n+1}^{-1} A^{T}\right)^{-1}, \quad Q=G_{n+1}^{-1} B^{T}\left(B G_{n+1}^{-1} B^{T}\right)^{-1} .
$$

- orthogonal iff in addition to (4), one has

$$
P^{T} G_{n+1} P=I \quad Q^{T} G_{n+1} Q=I
$$

and in this case $P$ and $Q$ are given by

- bi-orthogonal iff

$$
P=G_{n+1}^{-1} A^{T}, \quad Q=G_{n+1}^{-1} B^{T} .
$$

$$
B P=0, \quad A Q=0 .
$$

One main difference with the classical multiresolution analysis framework, is that the scaling functions $\left(\varphi_{j}^{n}\right)$ are not the result of a subdivision scheme. They are not fixed by the choice of the coefficients $\left(p_{i j}^{n}\right)$, and this is the reason why the Gram-Schmidt matrix $G_{n+1}$ appears in the orthogonality equations.

\subsection{Analysis and reconstruction algorithms}

Given an approximation $f_{n+1}=\sum_{i} x_{i}^{n+1} \varphi_{i}^{n+1}$ of $f$ in $V_{n+1}$, the next coarser approximation $f_{n}=\sum_{i} x_{i}^{n} \varphi_{i}^{n}$ and the details $g_{n}=\sum_{i} y_{i}^{n} \psi_{i}^{n}$ are computed by

$$
\left(x_{i}^{n}\right)=A\left(x_{i}^{n+1}\right) \quad\left(y_{i}^{n}\right)=B\left(x_{i}^{n+1}\right) .
$$

The inverse transformation is given by

$$
\left(x_{i}^{n+1}\right)=P\left(x_{i}^{n}\right)+Q\left(y_{i}^{n}\right) .
$$

The analysis and reconstruction formulas (6) and (7) are the same as in the case when the nested condition $V_{n} \subset V_{n+1}$ is satisfied, but they are related now to an approximation in two steps:

$$
\text { Analysis: } f_{n+1} \stackrel{\text { approx. }}{\longrightarrow} \tilde{f}_{n}=\sum_{i} x_{i}^{n} \widetilde{\varphi}_{i}^{n} \stackrel{\text { isomorph. }}{\longleftrightarrow} f_{n}=\sum_{i} x_{i}^{n} \varphi_{i}^{n}
$$

$g_{n}$

Figure 1 gives a geometric interpretation of one step of analysis and synthesis in the classical and new multiresolution framework, for the semi-orthogonal case.

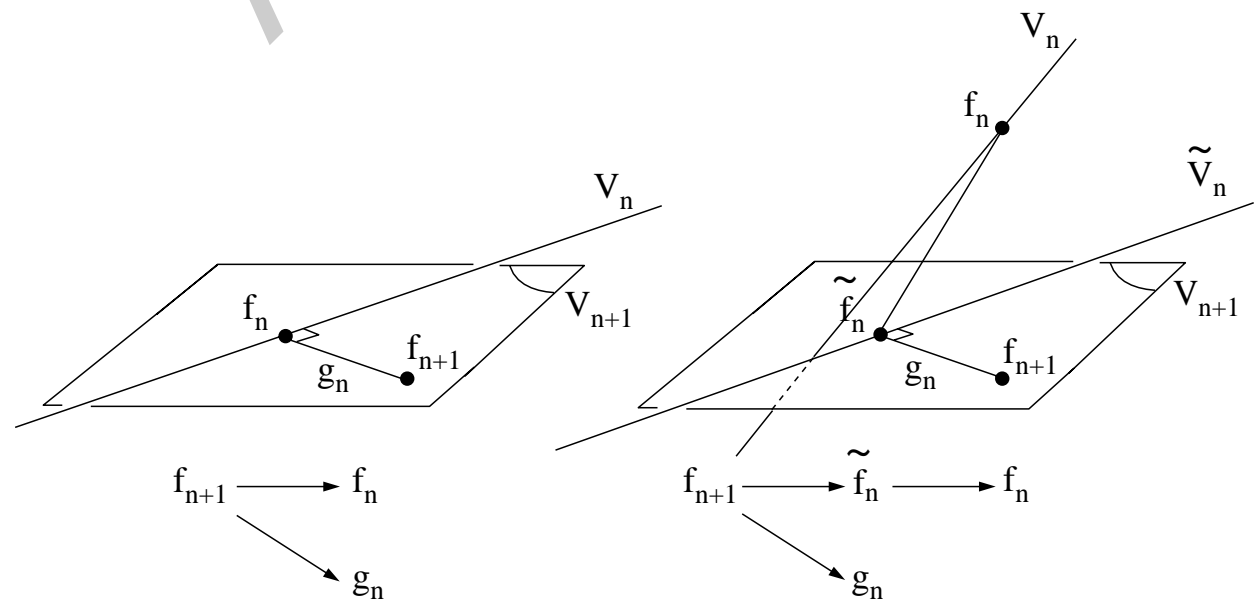

Figure 1: The classical and new multiresolution framework (semi-orthogonal case). 


\subsection{Choice of the intermediate functions $\tilde{\varphi}_{j}^{n}$}

A crucial point is the choice of the intermediate functions $\tilde{\varphi}_{j}^{n}$ (i.e. the choice of the matrix $P$ for the approximated refinement equation). In Section 3, this choice depends on the application, since $\tilde{\varphi}_{j}^{n}$ is chosen to be close to $\varphi_{j}^{n}$, and so that the approximated refinement equation (3) blends between the Haar and linear refinement equations. In Section 4, the choice of $\tilde{\varphi}_{j}^{n}$ is independent of the application, since it is chosen so that $f_{n}$ is always the best $L_{2}$-approximation of $f_{n+1}$ in $V_{n}$. This is achieved iff the smooth coefficients $\left(x_{i}^{n}\right)$ are the solution of the following linear system:

$$
G_{n}\left(x_{i}^{n}\right)_{i}=\left(<\varphi_{i}^{n}, \sum_{j} x_{j}^{n+1} \varphi_{j}^{n+1}>\right)_{i}
$$

and this implies that

$$
A=G_{n}^{-1}\left(<\varphi_{i}^{n}, \varphi_{j}^{n+1}>\right)_{i, j} .
$$

Since in Section 4, the multiresolution analysis verifies the semi-orthogonality conditions (4), the matrix $P$ (and hence the functions $\tilde{\varphi}_{j}^{n}$ ) are fixed by (5).

Therefore in the semi-orthogonal case, the choice (8) for the matrix $A$ and (5) for the matrix $P$ implies that $f_{n}$ is always the best approximation of $f_{n+1}$ in $V_{n}$. Nevertheless, since the sequence of approximation spaces $V_{n}$ is not nested, this best approximation property does not hold anymore between $f_{n}$ and $f_{n+k}$ if $k>1$. This implies theoretically that very coarse approximations could be far away from best approximations of the original data, and thereby inducing stability problems during the analysis. For the two following applications of sections 3 and 4, we didn't encount such stability problems.

In conclusion, this section has introduced a theoretical framework, that can be used to handle practical situations where the scaling functions arising naturally from the application do not fulfill any refinement equation.

\section{BLaC Wavelets}

In this section, the previous results are applied to define a one-parameter family of wavelets that perform a blend between Haar wavelets and linear wavelets. The purpose of BLaC wavelets is to find a compromise between the locality of the analysis (which is perfect for the Haar wavelets) and the regularity of the approximations (which is better for the linear wavelets). Therefore BLaC stands for Blending of Linear and Constant. More details on the motivation for the definition of BLaC wavelets can be found in [1].

In Section 3.1 the BLaC scaling functions and the approximated refinement equation, as defined in the previous section, are introduced. In Section 3.2, the BLaC wavelets, assuming semi-orthogonality and minimal support, are computed. The BLaC scaling and wavelet functions, and the approximated refinement equations depend on a blending parameter $\Delta \in[0,1]$, and are calculated so that for $\Delta=0$, the MRA scheme is equal to the Haar scheme, and for $\Delta=1$, to the linear scheme.

\subsection{BLaC scaling functions and approximated refinement equation}

The BLaC scaling function $\varphi_{i}^{n}$ is built by dilation by a factor $2^{n}$ and translation of $\left(i 2^{-n}\right)$ of the function $\varphi$ shown in figure $2: \varphi_{i}^{n}(x)=\varphi\left(2^{n}\left(x+i 2^{-n}\right)\right)$.

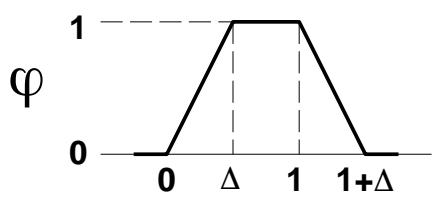

Figure 2: BLaC scaling function 
Since we want our algorithms to work on data defined on bounded domains, we select the indices $-1, \ldots, 2^{n}$, and take the restriction to the interval $[0,1]$ of the first and last scaling functions, $\varphi_{-1}^{n}$ and $\varphi_{2}^{n}$. Figure 3 shows the scaling functions at the level 2 of resolution. The lack of symmetry between the first and last scaling functions is due to the fact that there are $2^{n}-1$ Haar scaling functions, and $2^{n}$ linear scaling functions whose support intersects the interval $[0,1]$.

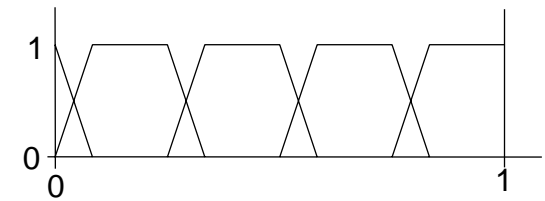

Figure 3: The BLaC scaling functions at level 2 of resolution.

From Figure 4 it is clear that $\varphi_{i}^{n}$ can not be written as a linear combination of the finer scaling functions $\varphi_{i}^{n+1}$ : in the dashed area, $\varphi_{i}^{n}$ has a slope, while all functions $\varphi_{i}^{n+1}$ are constant. Thus the new multiresolution analysis framework defined in the previous section has to be applied.

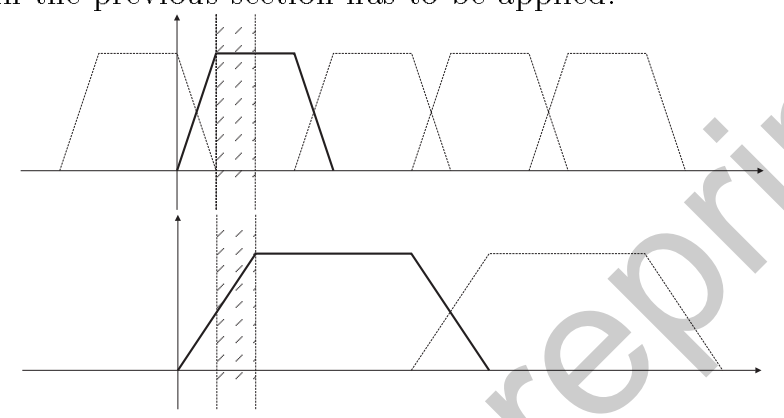

Figure 4: Need for non-nested approximating spaces.

The intermediate functions $\tilde{\varphi}_{i}^{n}$ must be chosen so that the approximated refinement equation (3) is a blending between the Haar and the linear refinement equations, and so that $\tilde{\varphi}_{i}^{n}$ is close to $\varphi_{i}^{n}$. For Haar scaling functions, the exact refinement equation is $\varphi_{i}^{n}=\varphi_{2 i}^{n+1}+\varphi_{2 i+1}^{n+1}$, and in the linear case it is $\varphi_{i}^{n}=\frac{1}{2} \varphi_{2 i}^{n+1}+\varphi_{2 i+1}^{n+1}+\frac{1}{2} \varphi_{2 i+2}^{n+1}$. Therefore we search for the following approximation of $\varphi_{i}^{n}$ :

$$
\begin{aligned}
& \tilde{\varphi}_{i}^{n}=\alpha \varphi_{2 i}^{n+1}+\varphi_{2 i+1}^{n+1}+(1-\alpha) \varphi_{2 i+2}^{n+1} \\
& \left\|\varphi_{i}^{n}-\tilde{\varphi}_{i}^{n}\right\|_{L^{2}([0,1])} \rightarrow \min .
\end{aligned}
$$

The constant $\alpha$ depends only on the blending parameter $\Delta$. As expected, $\alpha$ is equal to 1 for $\Delta=0$ and is equal to $1 / 2$ for $\Delta=1$, leading respectively to the Haar and linear refinement equations. Figure 5 illustrates the approximated refinement equation.

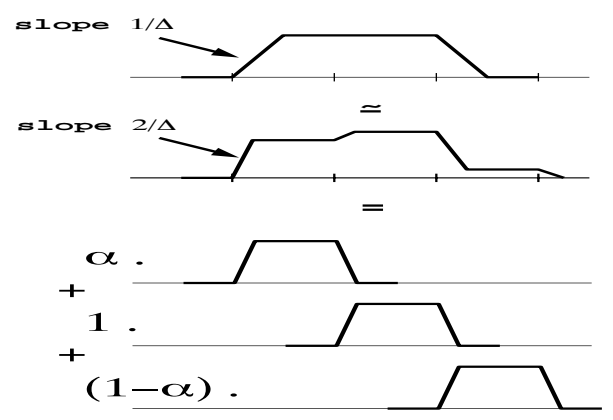

Figure 5: BLaC refinement equation

On the boundaries of the interval, the first and last approximated refinement equations are given by

$$
\begin{aligned}
\varphi_{-1}^{n} & \leftrightarrow \tilde{\varphi}_{-1}^{n}=\varphi_{-1}^{n+1}+(1-\alpha) \varphi_{0}^{n+1} \\
\varphi_{2^{n}-1}^{n} & \leftrightarrow \tilde{\varphi}_{2^{n}-1}^{n}=\alpha \varphi_{2^{n+1}-2}^{n+1}+\varphi_{2^{n+1}-1}^{n+1} .
\end{aligned}
$$


The matrix $P$ is now fixed as

$$
P=\left[\begin{array}{ccccc}
1 & 1-\alpha & & & \\
\alpha & 1 & 1-\alpha & & 0 \\
& \ddots & \ddots & \ddots & \\
0 & & \alpha & 1 & 1-\alpha \\
& & & \alpha & 1
\end{array}\right] .
$$

\subsection{BLaC wavelet functions}

We impose two constraints on the $\mathrm{BLaC}$ wavelet functions $\left(\psi_{i}^{n}\right)$ : they must be orthogonal to all intermediate functions $\left(\tilde{\varphi}_{i}^{n}\right)$ (i.e. the semi-orthogonality condition (4) must hold) and they must have minimal support. $\psi_{i}^{n}$ is a linear combination of the scaling functions $\left(\varphi_{j}^{n+1}\right)_{j}$. Adding a function $\varphi_{j}^{n+1}$ in this linear combination gives one more degree of freedom to determine the wavelet, but also increases the support of the wavelet. The minimal degree of freedom necessary to ensure the semi-orthogonality is 5, i.e.:

$$
\psi_{i}^{n}=a \varphi_{2 i}^{n+1}+b \varphi_{2 i+1}^{n+1}+c \varphi_{2 i+2}^{n+1}+d \varphi_{2 i+3}^{n+1}+e \varphi_{2 i+4}^{n+1}
$$

Indeed, in this case the support of the function $\psi_{i}^{n}$ intersects only the support of four basis functions of $\tilde{V}_{n}$, and the semi-orthogonality condition reduces to:

$$
<\psi_{i}^{n}, \tilde{\varphi}_{j}^{n}>=0 \quad j=i-1, i, i+1, i+2
$$

(10) is a linear homogeneous system of four equations in the five unknowns $a, b, c, d, e$. It is uniquely solved if we impose the $L_{2}$-norm of the wavelet to be equal to $1: \int\left|\psi_{i}^{n}\right|^{2}=1$. Note that the coefficients $a, b, c, d, e$ are also independent of the resolution level $n$. Figure 6 shows one BLaC wavelet for various values of $\Delta$.
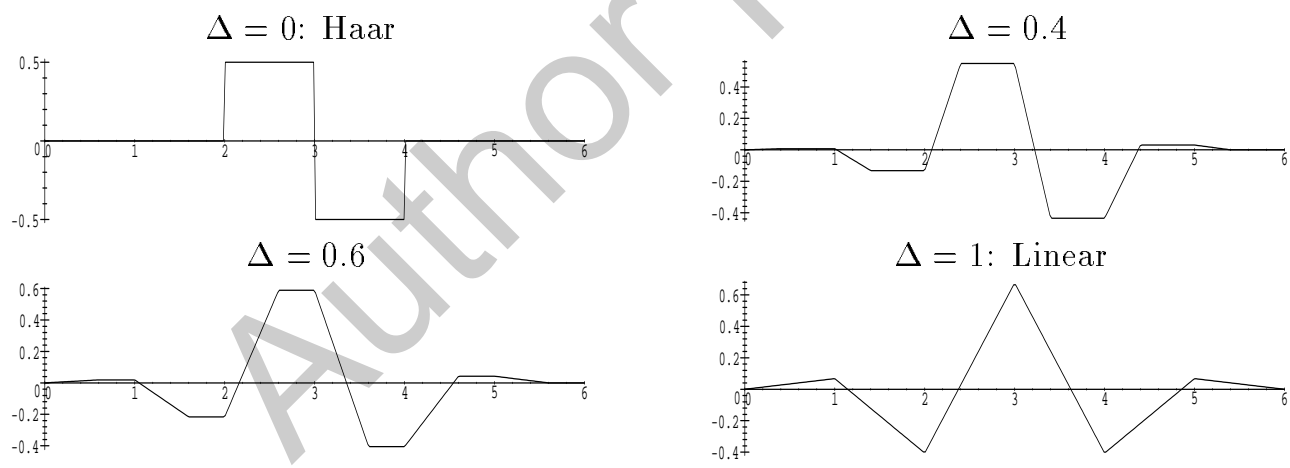

Figure 6: The BLaC Wavelets

On the boundaries of the interval, the first and last wavelets are linear combinations of only four scaling functions:

$$
\begin{aligned}
& \psi_{-1}^{n}=b_{0} \varphi_{-1}^{n+1}+c_{0} \varphi_{0}^{n+1}+d_{0} \varphi_{1}^{n+1}+e_{0} \varphi_{2}^{n+1} \\
& \psi_{2^{n}}^{n}=a_{1} \varphi_{2^{n+1}-4}^{n+1}+b_{1} \varphi_{2^{n+1}-3}^{n+1}+c_{1} \varphi_{2^{n+1}-2}^{n+1}+d_{1} \varphi_{2^{n+1}-1}^{n+1}
\end{aligned}
$$

$b_{0}, c_{0}, d_{0}, e_{0}, a_{1}, b_{1}, c_{1}, d_{1}$ are calculated by inverting the following linear homogeneous systems

$$
\begin{array}{ll}
<\psi_{-1}^{n}, \tilde{\varphi}_{j}^{n}>=0 & j=-1,0,1 \\
<\psi_{2^{n}}^{n}, \tilde{\varphi}_{j}^{n}>=0 & j=2^{n}-3,2^{n}-2,2^{n}-1,
\end{array}
$$

and normalizing the wavelets

$$
\int_{0}^{1}\left|\psi_{-1}\right|^{2}=\int_{0}^{1}\left|\psi_{2^{n}}\right|^{2}=1
$$


The matrix $Q$ is now fixed as

$$
Q=\left[\begin{array}{ccccccc}
b_{0} & c_{0} & d_{0} & e_{0} & & & \\
a & b & c & d & e & & 0 \\
& \ddots & \ddots & \ddots & \ddots & \ddots & \\
0 & & a & b & c & d & e \\
& & & a_{1} & b_{1} & c_{1} & d_{1}
\end{array}\right]
$$

and the semi-orthogonality condition $P^{T} G_{n+1} Q=0$ holds.

\subsection{Examples}

Figure 7 shows the result of the partial reconstruction of a 2D $(512 \times 512)$ data set, with $1 \%$ of the detail coefficients, for three different values of $\Delta$. On the top left is the original data. On the top right we used $\Delta=0$ (i.e Haar analysis), on the bottom left $\Delta=0.5$, and on the bottom right $\Delta=1$ (i.e. linear analysis) was used.

Because linear wavelets are more regular than Haar wavelets, the result at the bottom right looks smoother. On the other hand, the partial reconstruction using Haar wavelets at the top right is more sharp. The result of the reconstruction with $\Delta=0.5$ at the bottom left clearly shows that BLaC wavelets enables to find a compromise between the regularity and the locality of the analysis, or analogously between the sharpness and the smoothness.
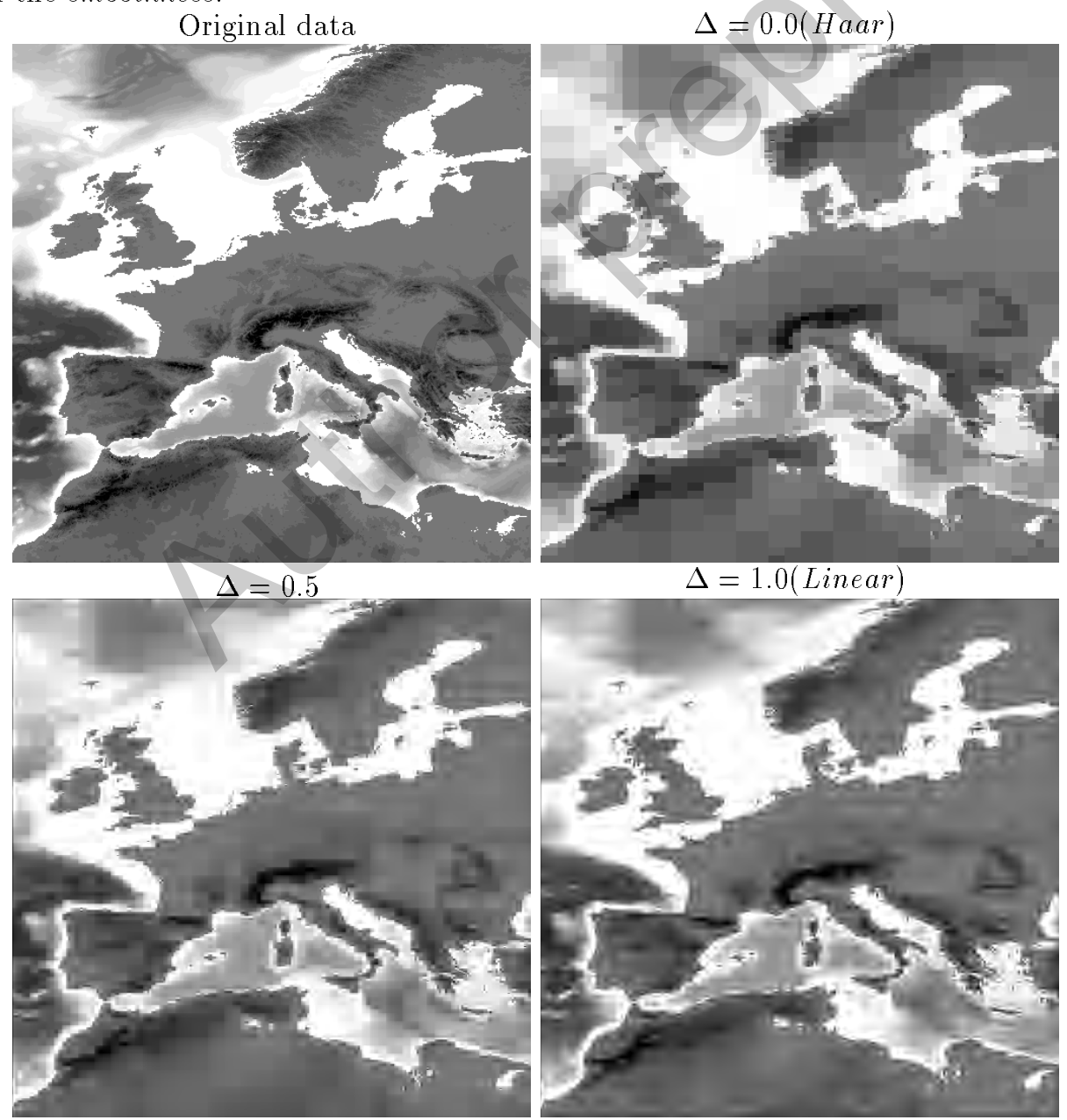

Figure 7: Partial reconstructions with BLaC wavelets.

(1\% of the coefficients) 


\section{Multiresolution analysis on hierarchical triangulations}

Large unstructured data sets originating from meteorological or geological measurements often need a special treatment because they are very expensive in storage and time. Although there exist a lot of different wavelet and multiresolution analysis constructions, they all are restricted to simple domains with subdivision connectivity. One exception is in [2], where the approach is to approximate the given domain by a simpler one which has subdivision connectivity.

For data defined on uniform grids, domains with subdivision connectivity are constructed by 2-split, 4 -split or 8 -split of intervals, squares or cubes. Previous approaches on multiresolution analysis of data defined on triangular meshes involve domains constructed by recursive 4 -split of triangles $[3,4,5]$.

The mathematical reason for the need of the subdivision connectivity for MRA relies in the need of a nested set of approximation spaces. Indeed, the nested set condition (1), or analogously the refinement equation implies that the support of each scaling function at one level of resolution must be the union of the supports of some finer scaling functions. Since for triangular multiresolution, the support of the scaling functions are the triangles of the mesh, it follows that the nested set condition (1) implies the subdivision connectivity of the mesh over which the data is defined. The development of Section 2 allows to construct multiresolution analysis on more general hierarchical triangulations, which don't have subdivision connectivity.

In Section 4.1. we describe the hierarchical triangulation structure that we chose for our multiresolution analysis. In Section 4.2. we explain how the theoretical framework introduced in Section 2 can be applied to find scaling and wavelet functions for such a hierarchical structure. The corresponding decomposition and reconstruction algorithms are introduced in Section 4.2. Examples are shown in Section 4.3. .

\subsection{The hierarchical triangulations and the approximation spaces}

The MRA scheme defined in the next section is dedicated to the analysis of piecewise constant functions defined on an irregular triangular planar mesh. Such functions can be the result of a finite element mesh calculus. At the finest level of resolution $N$, the domain $\Omega$ is the disjoint union of the triangles $T_{N, k}$ :

$$
\Omega=\bigcup_{k \in K(N)} T_{N, k}
$$

and the finest approximation space $V_{n}$ is the set of functions defined on $\Omega$ and constant over the triangles $T_{N, k}$.

The triangulation of the domain at the level $n$ is obtained by removing vertices from the triangulation at the level $n+1$ and re-triangulating the region equal to all triangles adjacent to the removed vertices. Such a construction defines disjoint polygonal regions $R_{n, j}$ in the domain $\Omega$, one for each set of adjacent removed vertices (see Figure 8):

$$
R_{n, j}=\bigcup_{k \in L(n+1, j)} T_{n+1, k}=\bigcup_{k \in L(n, j)} T_{n, k} .
$$




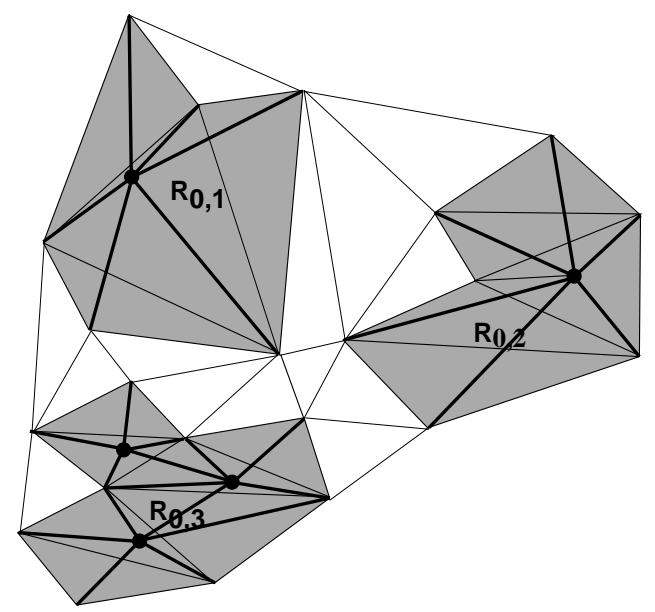

Figure 8: Polygonal regions defined by vertices removal.

The triangle $T_{n, k}$ are called the parent triangles of $R_{n, j}$, and the triangles $T_{n+1, k}$ are called child triangles of $R_{n, j}$.

At the level of resolution $n$, the approximation space $V_{n}$ is the set of functions defined on $\Omega$ and constant on the triangles $T_{n, k}$.

No assumption is made on the order of suppression of the vertices, and on the algorithm for retriangulating the polygonal region. In particular this paper does not address the problem of choosing an order of suppression of the vertices. It assumes that this order is known. This paper solves the problem of defining scaling and wavelet functions, together with smooth and detail coefficients, based on the knowledge of the hierarchy of triangulations.

In the current implementation the hierarchy is encoded with a graph structure. This graph has $N$ levels, one for each level of resolution (i.e. one for each triangulation of the domain $\Omega$ ). The nodes of the graph at the level $n$ are the polygonal regions $R_{n, j}$. Each node $R_{n, j}$ contains two main lists of pointers: one for the child triangles $T_{n+1, k}$, and one for the parent triangles $T_{n, k}$. Furthermore, a node $R_{n, j}$ points onto a node $R_{m, j^{\prime}}$ at a coarser level $m \leq n$, if $R_{m, j}$ contains a child triangle which is a parent triangle of $R_{n, j}$ (see Figure 9).

In the special case where the successive triangulations are constructed by 4 split, then each node has only one parent triangle and four child triangles, and the graph structure reduces to a forest of triangle quadtrees (like the one used in [5].
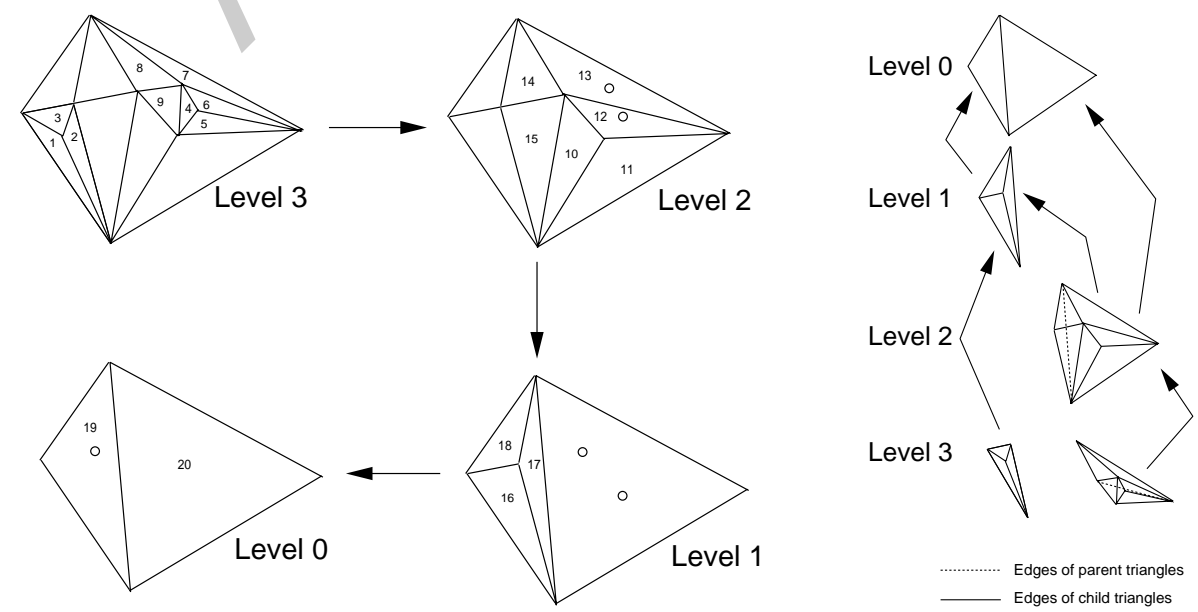

Figure 9: The hierarchical triangulations and the graph structure. 


\subsection{Analysis and synthesis matrices}

The scaling function $\varphi_{k}^{n}$ is the function equal to 1 on the triangle $T_{n, k}$, and 0 otherwise:

$$
\varphi_{k}^{n}=\chi_{T_{n, k}}
$$

Outside the polygonal regions $R_{n, j}$ defined in Section 4.1, the multiresolution analysis does nothing between level $n$ and $n+1$ : the scaling functions $\varphi_{k}^{n}, \varphi_{k}^{n+1}$ are the same, the smooth coefficients $\left(x_{k}^{n}\right),\left(x_{k}^{n+1}\right)$ also, and no detail coefficients need to be generated. It is therefore sufficient to compute the analysis and synthesis matrices $P, Q, A, B$ locally to each region $R_{n, j}$.

Let

$$
R_{n, j}=\bigcup_{k \in L(n+1, j)} T_{n+1, k}=\bigcup_{k \in L(n, j)} T_{n, k}
$$

be such a region.

$A$ is first computed, so that $f_{n}$ is always the best $L_{2}$-approximation of $f_{n+1}$ in $V_{n}$, as explained in Section 2.5: $A=G_{n}^{-1}<\varphi_{i}^{n}, \varphi_{j}^{n+1}>. G_{n}$ is here a diagonal matrix whose $i$-th diagonal element is the area of the triangle $T_{n, i}$, and $\left\langle\varphi_{i}^{n}, \varphi_{j}^{n+1}\right\rangle$ is equal to the area of the intersection of the triangles $T_{n, i}$ and $T_{n+1, j}$. Thus $A$ is the following $(\# L(n+1, j) \times \# L(n, j))$ matrix:

$$
A=\left(\frac{\operatorname{area}\left(T_{n, i} \cap T_{n+1, j}\right)}{\operatorname{area}\left(T_{n, i}\right)}\right)
$$

In the simple 4 split case, $A$ is the row matrix $\left(\frac{1}{4}, \frac{1}{4}, \frac{1}{4}, \frac{1}{4}\right)$.

$B$ is calculated so that the dual scaling and wavelet functions are orthogonal:

$$
A G_{n+1}^{-1} B^{T}=0
$$

and so that the dual wavelet functions are orthonormal:

$$
B G_{n+1}^{-1} B^{T}=I .
$$

This is done in two steps: first fix a submatrix of dimension $\# L(n, j) \times \# L(n, j)$ of $B$ to be the identity and solve (11). Then apply a Gram-Schmidt algorithm to orthonormalize the rows of $B$.

This choice for the analysis matrices $A$ and $B$ ensures that

- the approximation $f_{n}$ at the level $n$ is the best $L_{2}$ approximation of $f_{n+1}$,

- the intermediate approximation $\tilde{f}_{n}$ at the level $n$ is also the best $L_{2}$ approximation of $f_{n+1}$.

Furthermore, this choice for the analysis matrices implies that the synthesis matrices $P$ and $Q$ can be calculated from $A$ and $B$ by

$$
P=G_{n+1}^{-1} A^{T}\left(A G_{n+1}^{-1} A^{T}\right)^{-1}, \quad Q=G_{n+1}^{-1} B T,
$$

where $G_{n+1}^{-1}$ is here the diagonal matrix with $i$-th diagonal element equal to the area of the triangle $T_{n+1, i}$.

Figure 10 shows the decomposition of a function $f_{n+1}$ in a region $R_{n, j}$ containing four child triangles $T_{n+1, k}$ and two parent triangles $T_{n, k}$. This region is created by the removal of one interior point. The decomposition is a two step process, as explained in Section 2.4: first apply the analysis matrices to find $\tilde{f}_{n}$ and the two wavelet components $y_{0}^{n} \psi_{0}^{n}, y_{1}^{n} \psi_{1}^{n}$. Then apply the isomorphism, i.e. replace the intermediate scaling functions $\tilde{\varphi}_{i}^{n}$ by the scaling functions $\varphi_{i}^{n}$. 


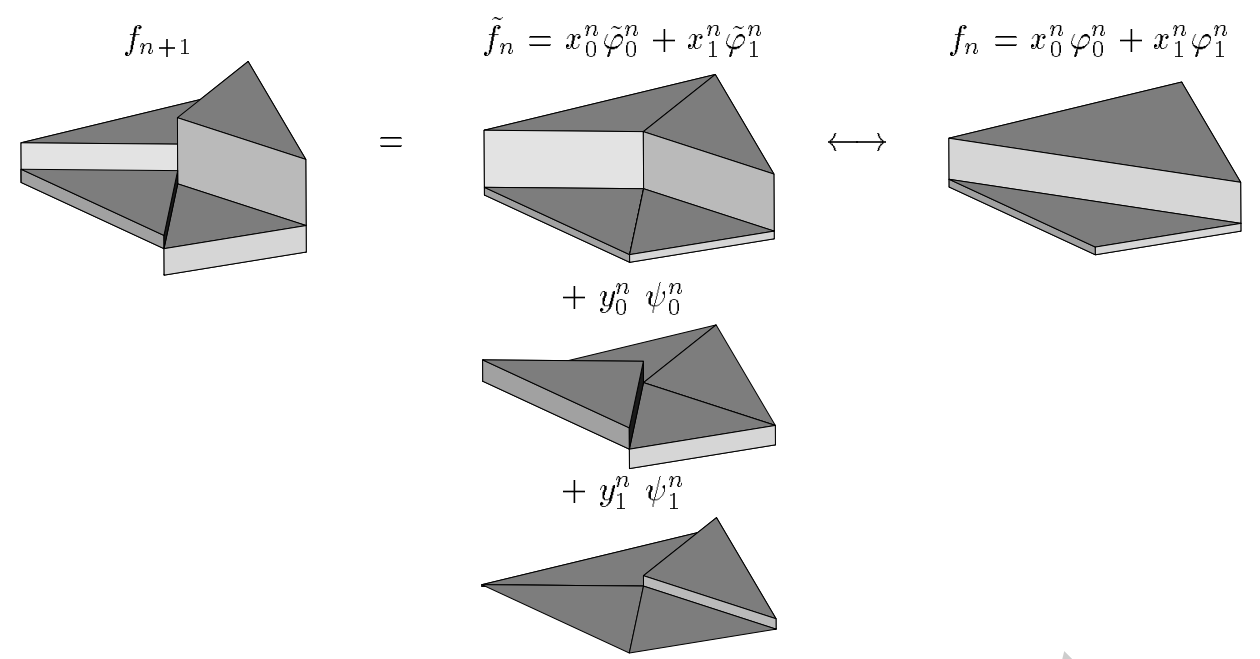

Figure 10: Triangular wavelet decomposition.

\subsection{Triangular decomposition and reconstruction algorithms}

The graph structure described in Section 4.1., and the analysis and synthesis matrices introduced in Section 4.2. enable to implement the three following algorithms.

- Algorithm 1: Triangular wavelet decomposition.

This algorithm starts with the values $\left(x_{i}^{N}\right)$ of a function $f$ on the finest triangles $T_{N, k}$. Then for each level of resolution $n$ and for each polygonal region $R_{n_{f}, j}$ (as defined in Section 4.1.), the analysis matrices $A$ and $B$ introduced in Section 4.2. are used to compute the smooth coefficients $\left(x_{i}^{n}\right)$ and the detail coefficients $\left(y_{i}^{n}\right)$. These coefficients are stored in memory in place of the corresponding finer scaling coefficients $\left(x_{i}^{n+1}\right)$.

At the end remains only the scaling coefficients $\left(x_{i}^{0}\right)$ corresponding to the approximation of $f$ at the lowest level of resolution, and the detail coefficients $\left(y_{i}^{n}\right)$ corresponding to all regions $\left(R_{n, j}\right)$.

- Algorithm 2: Reconstruction at a fixed level $n$.

This algorithm starts with the output of the wavelet decomposition algorithm. For each level of resolution $k$ lower than $n$, and for each region $R_{k, j}$, the synthesis matrices $P$ and $Q$ are used to recover the scaling coefficients $\left(x_{i}^{k+1}\right)$ from the coarser coefficients $\left(x_{i}^{k}\right)$ and the details $\left(y_{i}^{k}\right)$.

- Algorithm 3: Threshold reconstruction.

This algorithm starts with the output of the wavelet decomposition algorithm, and with a threshold value $\epsilon$. This algorithm select the subgraph of the graph structure introduced in Section 4.1., which contains all regions $R_{n, j}$ that have an associated detail coefficient whose absolute value is greater than the threshold value $\epsilon$. Then the synthesis matrices $P$ and $Q$ are applied on all these selected regions to recover the scaling coefficients $\left(x_{i}^{n+1}\right)$ from the coarser coefficients $\left(x_{i}^{n}\right)$ and detail coefficients $\left(y_{i}^{n}\right)$ that have an absolute value greater than $\epsilon$.

\subsection{Examples}

The hierarchical triangulations corresponding to the examples of Figure 11 were obtained by inserting randomly chosen vertices in the square domain $\Omega=[0,512] \times[0,512]$, and constructing their Delaunay triangulation. The coarsest triangulation of the domain $\Omega$ consists of two triangles covering it. The values of the test function at the finest level of resolution are the mean values of a $512 \times 512$ image on the finest triangles. 
The top picture of Figure 11 shows the original data, consisting of 48682 triangles together with their function value (a gray level between 0 . and 256.).

The middle left picture of Figure 11 shows the result of the reconstruction of the data at a fixed level (algorithm 2 of Section 4.3.), using approximatively $40 \%$ of the detail coefficients. The bottom left picture shows the corresponding triangulation.

The middle right picture shows the result of a threshold reconstruction (algorithm 3 of Section 4.3.) using approximatively the same number of detail coefficients. The corresponding triangulation (bottom right picture) illustrates that this algorithm selects more vertices in the areas of strong variations of the test function.
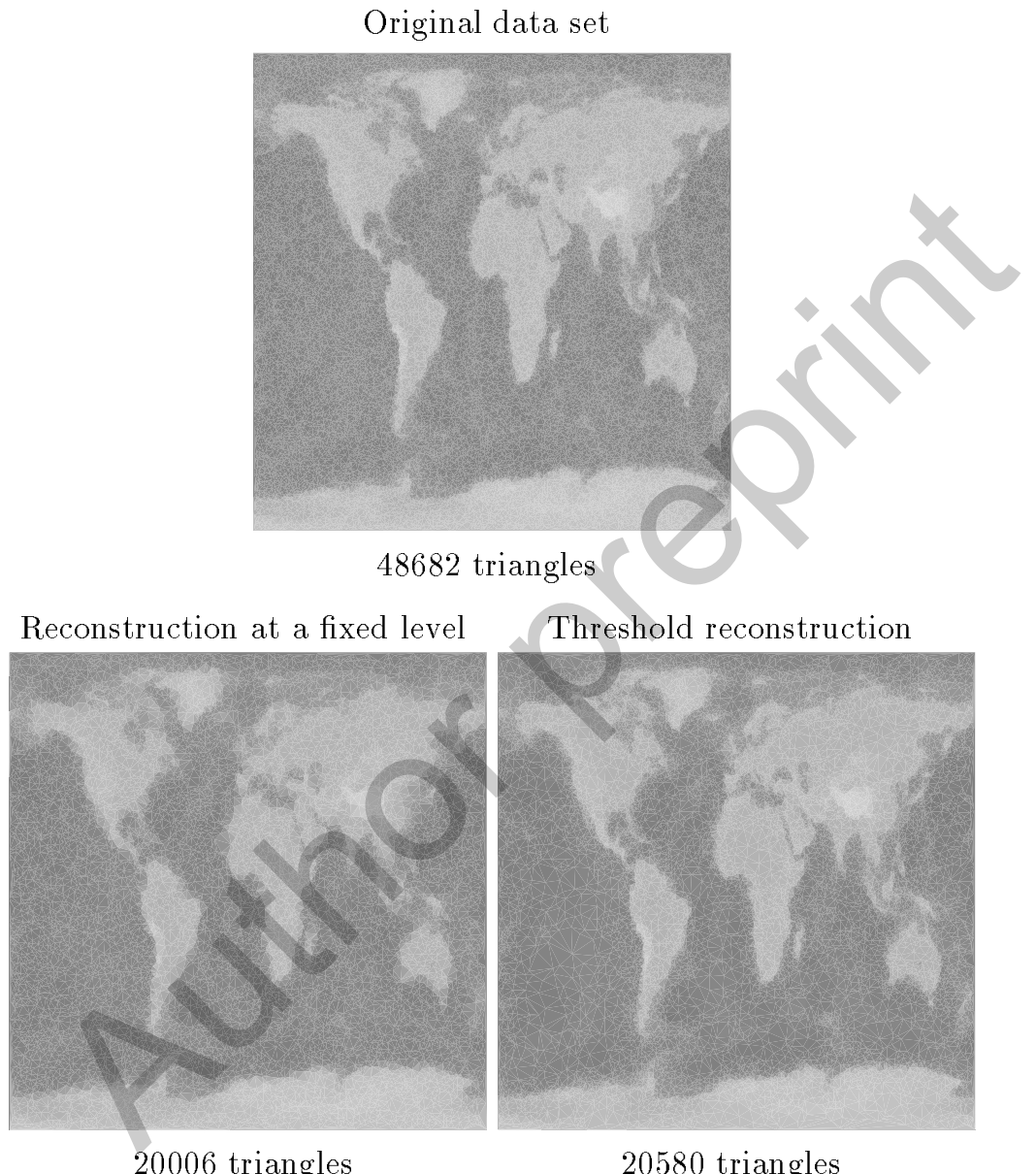

Threshold reconstruction
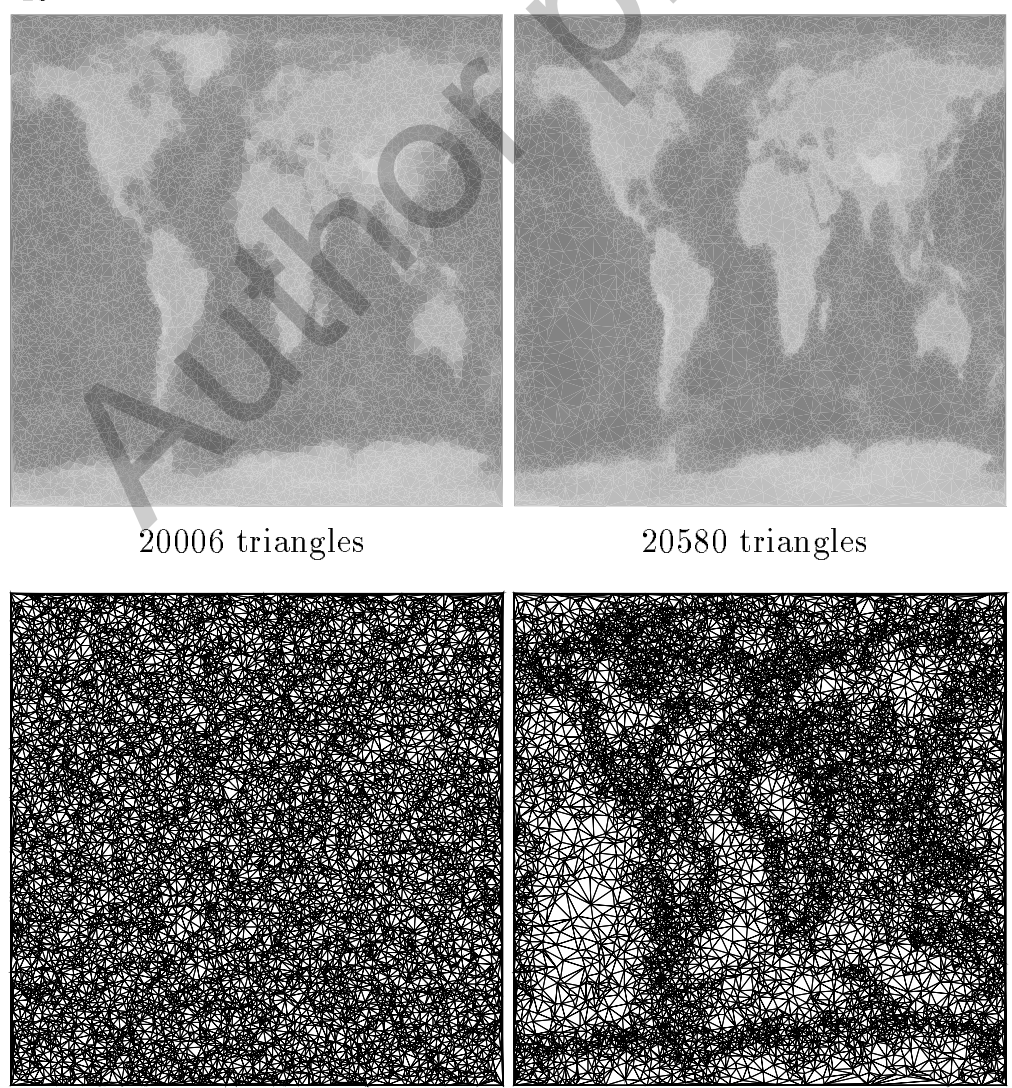

Figure 11: Partial reconstructions. 


\section{References}

1. Bonneau G.P., Hahmann S., Nielson G.M. BLaC Wavelets: a multiresolution analysis with non-nested spaces, Proceedings of the VIS'96, ACM, New York, 1996, pp 43-48.

2. Eck M., DeRose T., et al., Multiresolution Analysis of Arbitrary Meshes, Proceedings of SIGGRAPH'95. ACM, New York, 1995, pp. 173-182.

3. Lounsbery J.M., Multiresolution analysis for surfaces of arbitrary topological type, PhD Thesis, Dept. of Computer Science and Engineering, U. of Washington, 1994.

4. Stollnitz E., DeRose T., Salesin D., "Wavelets for Computer Graphics, Theory and Applications", Morgan Kaufman, 1996.

5. Schröder P., Sweldens W., Spherical wavelets: Efficiently representing functions on the sphere, Proceedings of SIGGRAPH'95, ACM, New York, 1995, pp 161-172. 